\title{
Saturating The Bekenstein-Hawking Entropy Bound With Initial Data Sets For Gravitational Collapse
}

\author{
Sina Bahram* \\ Department of Physics, Cornell University
}

(Dated: June 6, 2022)

\begin{abstract}
It is possible to find initial states for gravitational collapse whose entropy approximately saturates the Bekenstein-Hawking entropy of the final black hole. The prototypical example of such a state is that envisaged by Zurek and Thorne, and also by Susskind: for a black hole of mass $M$, a number $\sim M^{2}$ of quanta with energies of order $\sim M^{-1}$ are accreted on a timescale of order $\sim M^{3}$, an approximate time-reverse of Hawking evaporation. There is lore that all initial states which saturate the Bekenstein-Hawking entropy must involve a formation timescale of this order, $\sim M^{3}$, and not the much shorter dynamical timescale $\sim M$. Counterexamples to this lore have been found by Sorkin, Wald and Zhang, and also by Hsu and Reeb, in the form of semiclassical initial data sets. However the spacetimes that correspond to these counterexamples possess white holes in the past, as well as black holes in the future, which casts doubt on their physical relevance. We modify the counterexamples to eliminate the white holes, yielding formation timescales of order $\sim M^{2}$, and argue that the lore is unfounded.
\end{abstract}

\section{INTRODUCTION}

Forty some years have passed since Bekenstein conjectured that a black hole is a thermal system with an entropy proportional to the area of its event horizon [1. Subsequent careful analyses by Hawking confirmed the thermal nature of black holes [2, 3]. Since then, the microphysical interpretation of the Bekenstein-Hawking entropy has been the subject of much speculation and discussion [4]. One of the earliest proposals was that black hole entropy counts the number of distinct initial data sets from which the black hole might have been formed [1, 2]. The motivation for this proposal is that the number of states of a system is not expected to change if the system undergoes a unitary quantum mechanical evolution. Thus, one expects that the number $N$ of distinct initial data sets that can collapse to form a given black hole must match its number of internal states, and correspondingly match the Bekenstein-Hawking entropy, $\ln N \sim M^{2}$, where $M$ is the black hole mass 1

As is well known, for black holes that are formed in astrophysical collapses, the entropies $S$ of the pre-collapse configurations do not saturate the Bekenstein-Hawking value. When gravity is weak, the maximum entropy that can be contained in a region of size $R$ using an energy $E$ is that of thermal radiation, $S \sim(E R)^{3 / 4}$, and for the final stages of collapse taking $E \sim M$ and $R \sim M$ gives $S \sim M^{3 / 2}$, far smaller than the Bekenstein-Hawking value. Although this estimate is based on the assumption of weak gravity which is violated during the late stages of collapse, one expects it to be a robust upper bound for systems which start in a weak gravity regime. The final stages of such collapses occur on a timescale $\sim M$.

\footnotetext{
* sb933@cornell.edu

1 Throughout this paper we work in the units of $k=c=\hbar=G=$ 1.
}

A different formation scenario can saturate 2 the Bekenstein-Hawking entropy. As first pointed out by Zurek and Thorne [7] (see also [8]) small black holes of initial mass $m$ much smaller than the final mass $M$ can accrete quanta of energies $E \sim 1 / m$ one-by-one, each taking a time of order $m$. The black hole then grows in an approximate time reverse of the Hawking evaporation process, yielding a final mass $M$ after accreting $\sim M^{2}$ quanta. The number $N$ of such initial states is then given by $\ln N \sim S \sim M^{2}$. However, this formation process occurs on a much longer timescale than astrophysical collapse, of order $\tau \sim M^{3}{ }^{3}$.

One can also construct scenarios which are intermediate between the two extremes of dynamical collapse and the Thorne-Zurek scenario. We consider a black hole accreting quanta of a quantum field where each sector $l, m$ for $l \leq l_{\max }$ is an independent $1+1$ dimensional thermal gas with quanta of energy $E \sim l_{\max } / M$ and size $\tau$. The total number of quanta per sector is $\tau E \sim \tau l_{\max } / M$, and the total number of quanta is given by multiplying by the number $l_{\max }^{2}$ of sectors, giving $S \sim \tau l_{\max }^{3} / M$. Similarly the total energy per sector is $\tau E^{2} \sim \tau l_{\max }^{2} / M^{2}$, and the total energy is then $\tau l_{\max }^{4} / M^{2}$. Equating this to $M$ and eliminating $l_{\max }$ yields a relation between entropy $S$, black hole mass $M$ and formation timescale which interpolates between the two extreme examples:

$$
S \sim M^{5 / 4} \tau^{1 / 4}
$$

The examples discussed above, and specifically the relation (1), all support a lore in the community that generic black hole microstates must be formed by collapse processes on timescales much longer than $M$. In

\footnotetext{
${ }^{2}$ In the weak sense that the logarithm of the number of states scales like $M^{2}$, not in the stronger sense that it is $M^{2} / 4$ plus subleading corrections.

3 Also see 9], which shares some similarities with the Thorne Zurek construction.
} 
particular, one might conjecture that the relation (1) is generic, valid in order of magnitudes for all collapse scenarios. The purpose of this paper is to investigate this lore and see if there is any evidence against it.

There have been a number of attempts to challenge the conjectured relation (1). Most famous among these attempts are the initial data sets first constructed by Sorkin et.al. in [10] which later became known as "monsters" [11] 4. A monster is a time symmetric and asymptotically flat spacelike Cauchy surface that is foliated by nearly marginally trapped 2-spheres. The stress energy tensor is everywhere vacuum except for a compact spherical region which is filled with a thermalized gas of radiation. Monsters are expected to form black holes within a timescale on the order of $M$. A large entropy on the order of $M^{2}$ is achieved for monsters due to the enhanced effect of the intrinsic curvature on the Cauchy surface. Specifically, the large intrinsic curvature allows for a relatively large number of quanta to be packed together in a spatial region with a radius on the order of the Schwarzschild radius 5 .

Despite the fact that monsters saturate the BekensteinHawking entropy bound as initial data sets, they may not give rise to valid gravitational collapse processes. In fact, it is far from clear if their full Cauchy development is pathology free. This question is particularly important since a monster, by virtue of being in the close vicinity of the future apparent horizon, is expected to be largely within the future event horizon. Thus, one would need to ascertain that the future event horizon is everywhere regular.

Moreover, monsters have a time symmetric Cauchy development. This implies that they are endowed with two distinct curvature singularities, a black hole and a white hole. White holes possess classical and quantum instabilities that render them unphysical [13 15]. These issues suggest that monster initial sets are not physically realistic or relevant counterexamples to the conjectured relation (1).

In this paper we investigate the global structure of the full Cauchy development of monster-like initial conditions. We shall focus on spacetimes with a dust dominated collapsing core. We review the essential geometric theory in Secs. IIIII A, and III B. In Sec. III C we show how a monster-like initial condition gives rise to a collapsing spacetime that saturates the Bekenstein-Hawking entropy bound and has an everywhere regular future event horizon. Finally, in Sec. IV we discuss a class of examples obtained by modifying the spacetime geometry in the past in order to get rid of the white hole singularity.

\footnotetext{
${ }^{4}$ Also see 10, 12 for the so-called bags of gold initial data sets. Similar to monsters, the full Cauchy development of these initial data sets involve both a white hole and a black hole singularity.

${ }^{5}$ In this case a spherical region with an area of $\sim M^{2}$ can have a volume $\sim M^{4}$, significantly larger than what it would be if the intrinsic curvature were negligible.
}

We show that the modified spacetimes satisfy the weak energy condition.

\section{THE HYDRODYNAMIC APPROXIMATION FOR DUST FLUIDS}

In this section we briefly review the basics of dust fluids and discuss the conditions for the validity of the hydrodynamic approximation in arbitrary spacetimes.

A pressureless dust fluid is characterized by a fourvelocity vector field $\vec{u}$ and a number density $n$, satisfying $u^{a} \nabla_{a} u^{b}=0$ and $\nabla_{a}\left(n u^{a}\right)=0$. The stress energy tensor for the dust fluid is given by

$$
T_{a b}=\rho u_{a} u_{b}=m n u_{a} u_{b},
$$

where $\rho=m n$ is the energy density measured in the comoving frame of the fluid, and $m$ is the particle mass. Here we allow $m$ to have dependence on spatial coordinates.

The entropy $S$ of a dust fluid is roughly equal to the number of its constituent particles. Therefore, one can associate a covariantly conserved entropy current $s^{a}=n u^{a}$ to the dust fluid 6 and then compute the total entropy on any achronal spacetime slice $\Sigma$ by

$$
S=\int_{\Sigma} s^{a} d \Sigma_{a}=\int_{\Sigma} \frac{\rho}{m} u^{a} d \Sigma_{a}=\int_{\Sigma} n u^{a} d \Sigma_{a} .
$$

The validity of the hydrodynamic approximation underlying the dust fluid model hinges on the following conditions 7 .

$$
\begin{aligned}
& (8 \pi m) n^{-1 / 3} \gtrsim 1, \\
& (8 \pi m)^{-1} \gtrsim 1, \\
& \left(\frac{16 \pi}{3}\right)^{1 / 3} \frac{m}{|\mathbf{D} m|} \gtrsim n^{-1 / 3}, \\
& \mathcal{L} \gg n^{-1 / 3}
\end{aligned}
$$

where $\mathbf{D}$ denotes differentiation with respect to the spatial coordinates, and $\mathcal{L}$ is the radius of curvature defined as $\mathcal{L}^{-4} \equiv R_{a b c d} R^{a b c d}$ for the spacetime Riemann curvature tensor $R_{a b c d}$. To have a valid model of gravitational collapse, one needs to ensure that these relations are valid everywhere to the past of the event horizon of the ensuing black hole.

The above conditions can be understood as follows. The first condition is the non-degeneracy condition, asserting that the degeneracy pressure can be ignored as long as the dust particles remain non-overlapping. The

\footnotetext{
6 the entropy current defined here is generally correct upto some order unity prefactor.

7 These relations are not precise inequalities. They are only defined upto factors of order unity. The numerical factors are added for convenience when we later discuss saturating these relations.
} 
violation of this condition undermines the pressureless dust description of the fluid ${ }^{8}$. The second condition asserts that the Compton wavelength of each particle must be above the Planck length 9 for quantum gravity effects to be ignored. The third and fourth conditions require the radius of curvature and the length scales over which the particle mass varies to be larger than the intraparticle spacing. This last condition is equivalent to saying that most of the contribution to the total entropy comes from the modes with wavelengths small compared to the spacetime radius of curvature.

\section{DUST OBJECTS IN SPHERICAL SYMMETRY}

\section{A. Lemaître-Tolman-Bondi dust models}

Consider a spherically symmetric compact object that is composed of non-interacting dust particles. For an arbitrary density profile ${ }^{10}$, the interior geometry of the object is described by the Lemaitre-Tolman-Bondi (LTB) metric [16 18

$$
d s^{2}=-d t^{2}+\frac{R^{\prime}(t, r)^{2} d r^{2}}{1-r^{2} k(r)}+R(t, r)^{2} d \Omega^{2},
$$

where prime denotes differentiation with respect to the coordinate $r, R(t, r)$ is the areal radius, $k(r)$ determines the intrinsic curvature on constant $t$ slices 11 , and $d \Omega^{2}=$ $d \theta^{2}+\sin \theta^{2} d \phi^{2}$. Since the metric (5) is describing the interior of a compact region, the coordinate radius $r$ is only defined out to some $r_{\text {out }}$. Furthermore, $R^{\prime}(t, r)>0$ and $k(r)<1 / r^{2}$ as the spherical shells are not allowed to cross one another.

Given the metric (5), the stress energy tensor for the pressureless dust fluid is given by (2) with

$$
\vec{u}=\vec{\partial}_{t}, \quad \rho(t, r)=\frac{\bar{\rho} r^{2}}{R(t, r)^{2} R^{\prime}(t, r)},
$$

for some positive constant $\bar{\rho}$. The metric function $R(t, r)$ can be solved for analytically from the $G_{r r}=8 \pi T_{r r}=0$

${ }^{8}$ As the intra-particle spacing approaches the Compton wavelength of particles, the quantum fluctuations in the stress energy tensor of the dust fluid become comparable to the classical stress energy tensor.

9 The Planck length is defined as $\sqrt{\hbar G / c^{3}}$, which is equal to unity in our choice of units.

10 For homogeneous density profiles, the metric is the FriedmannRobertson-Walker (FRW) metric

$$
d s^{2}=-d t^{2}+a(t)^{2}\left[\frac{d r^{2}}{1-\kappa r^{2}}+r^{2} d \Omega^{2}\right]
$$

where $\kappa$ is a constant taking $0, \pm 1$ values. The areal radius $R(t, r)$ in the metric (5) then reduces to $r a(t)$ in the homogeneous case.

11 The intrinsic scalar curvature of the constant $t$ slices is ${ }^{(3)} R=$ $6 k(r)+2 r k^{\prime}(r)$. component of the Einstein's equations. For the reason mentioned in footnote 5 , we will restrict attention to the $k(r)>0$ case. In this case we have

$$
G_{r r}=0 \Rightarrow \dot{R}(t, r)^{2}+2 R(t, r) \ddot{R}(t, r)+r^{2} k(r)=0,
$$

where dot denotes differentiation with respect to $t$. The closed form solution to Eq. (7) is given by the parametric equations

$R(t, r)=\frac{4 \pi \bar{\rho} r}{3 k(r)}(1-\cos u), \quad t-t_{0}(r)=\frac{4 \pi \bar{\rho}}{3 k(r)^{3 / 2}}(u-\sin u)$,

where $0 \leq u \leq 2 \pi$. Evidently, there are two curvature singularities in this spacetime. The function $t_{0}(r)$ is the "bang function" specifying the coordinate time at which a spherical shell at a coordinate radius $r$ departs from one of the curvature singularities, only to arrive at the other one at the coordinate time $t=t_{0}(r)+4 \pi^{2} \bar{\rho} /\left[3 k(r)^{3 / 2}\right]$. Note that the function $t_{0}(r)$ is chosen such that the inner spherical shells arrive at the curvature singularities prior to the outer spherical shells.

\section{B. Dust objects with vacuum exterior}

In this section we construct models of collapsing dust objects by gluing LTB interior solutions to Schwarzschild exterior solutions.

As we mentioned in Sec. III A, the LTB metric describes the interior geometry of a compact spherical region. The boundary of the interior region is a timelike three dimensional hypersurface that consists of points with the LTB coordinates $\left\{t, r_{\text {out }}\right\}$.

The exterior geometry is given by the Schwarzschild metric

$$
d s^{2}=-\omega(\bar{r}) d \bar{t}^{2}+\omega(\bar{r})^{-1} d \bar{r}^{2}+\bar{r}^{2} d \Omega^{2}, \quad \omega(\bar{r})=1-\frac{2 M}{\bar{r}},
$$

where $M$ is the gravitational mass associated with the collapsing object. The boundary of the compact inner region in the Schwarzschild coordinates is given by $\left\{t, R\left(t, r_{\text {out }}\right)\right\}$, where $t$ is the proper time of the freely falling dust particles on the boundary of the inner region. Note that $t$ coincides with the LTB time coordinate.

To smoothly join inner LTB solutions to outer Schwarzschild solutions one must impose the Israel junction conditions on the boundary. The details of this process is essentially identical to the Oppenheimer-Snyder collapse and it can be found in many references [e.g. see [19]. It follows from imposing the Israel junction conditions on the boundary region that

$$
\begin{aligned}
& M=\frac{R\left(t, r_{\text {out }}\right)}{2}\left[\dot{R}\left(t, r_{\text {out }}\right)^{2}+r_{\text {out }}^{2} k\left(r_{\text {out }}\right)\right] \\
& =\frac{4 \pi \bar{\rho} r_{\text {out }}^{3}}{3}
\end{aligned}
$$

where the second equality above follows from Eqs. (7) and (8). Actually, a more general statement follows from 
Eq. 7); that the Misner-Sharp mass enclosed by each spherical shell at the LTB coordinate radius $r$ is

$$
M(r)=\frac{R(t, r)}{2}\left[\dot{R}(t, r)^{2}+r^{2} k(r)\right]=\frac{4 \pi \bar{\rho} r^{3}}{3} .
$$

The future (and past) apparent horizon is then defined as the surface for which 1213

$$
1-\frac{2 M(r)}{R(t, r)}=1-r^{2} k(r)-\dot{R}(t, r)^{2}=0
$$

for all $r$. All spherical shells are said to be marginally trapped on the apparent horizon. Once the apparent horizon is crossed, i.e.

$$
1-\frac{2 M(r)}{R(t, r)}=1-r^{2} k(r)-\dot{R}(t, r)^{2}<0
$$

for some spherical shell at $r$, then the shell is said to have become trapped.

\section{Dust objects with order $M^{2}$ entropy}

In this section we show that one can construct collapsing dust objects using the LTB geometries with $k(r)>0$ that come close to saturating the Bekenstein-Hawking entropy bound of $S_{\mathrm{BH}}=4 \pi M^{2}$ for non-black hole objects 14

Using Eqs. (3) 15 (5) (for $k(r)>0$ ) and (6) we find the entropy of a collapsing dust object on an achronal but otherwise arbitrary slice to be

$$
S=4 \pi \int_{0}^{r_{\text {out }}} d r \frac{\bar{\rho} r^{2}}{m(r) \sqrt{1-r^{2} k(r)}} .
$$

Clearly, not all choices of the metric and particle mass functions result in valid models that can saturate the Bekenstein-Hawking entropy bound. In addition to requiring the hydrodynamic relations (4) to remain valid

12 This can also be derived by finding the necessary condition for which the congruences of the outgoing future and past directed null geodesics that are orthogonal to a 2 -sphere at the coordinate radius $r$ have zero expansions.

13 It is proven in [20] that for strongly predictable spacetimes satisfying the weak or strong energy conditions the future apparent horizon is inside the future event horizon. The LTBSchwarzschild spacetimes for which the future event horizon is regular everywhere are strongly predictable and satisfy both the weak and strong energy conditions.

14 This cannot be done for homogeneous dust objects in the Oppenheimer-Snyder collapse. The entropy in these models is roughly $M / m$. However, for the fluid to obey the first and fourth of the conditions given in 4 we must have $m^{-1} \lesssim n^{-1 / 3} \ll M$. Similar conclusions hold for the LTB geometries with $k(r)=0$ or $k(r)<0$.

15 Note that the LTB metric (5) together with Eq. (8) guarantee $\nabla_{a}\left(\rho u^{a} / m\right)=0$ for $m$ being an arbitrary function of $r$. everywhere to the past of the future event horizon 16 we must also require most of the future apparent horizon to consist of 2 -spheres for which $|\dot{R}(t, r)| \ll 1$. This is a revision of a similar requirement that was originally set forth in 10]. It can be seen from Eq. $\sqrt{12}$ that the future (or past) apparent horizon cannot be entirely foliated by 2 -spheres with $\dot{R}=0$. In fact that would require $k(r)=1 / r^{2}$ for all $r$, for which the metric (5) would not be defined. Nonetheless, one might wonder if there are any constraints on how small $|\dot{R}|$ is for the 2 -spheres that foliate the apparent horizon. Without any constraints, the integral (14) can be made arbitrarily large for choices of $k(r)$ that are arbitrarily close to $1 / r^{2}$.

A physically realistic choice of constraint on the smallness of $|\dot{R}|$ along the apparent horizon can be explained as follows. Consider the 3-dimensional achronal surface $\Sigma$ parametrized by $t_{\Sigma}(r)$ that is foliated by the 2 -spheres with $\dot{R}=0$. Now consider a constant $t$ surface that intersects $\Sigma$ at $\left\{t_{\Sigma}(\check{r}), \check{r}\right\}$. Define a coordinate radius $\tilde{r}$ on this constant $t$ surface using the following relation,

$$
R\left[t_{\Sigma}(\check{r}), \tilde{r}\right]=2 M(\check{r})
$$

The proper distance between the shell at $\check{r}$ and the shell at $\tilde{r}$ on this constant $t$ surface is given by

$$
\int_{\tilde{r}}^{\check{r}} \sqrt{g_{r r}} d r .
$$

This distance corresponds to the spatial distance that the shell at $\left\{t_{\Sigma}(\check{r}), \check{r}\right\}$ would have to travel in order to arrive at the apparent horizon. We require this distance to be larger than the particle spacing on the shell at $\check{r}$, i.e.

$$
\int_{\tilde{r}}^{\check{r}} \sqrt{g_{r r}} d r \geq 4 n^{-1 / 3}\left[t_{\Sigma}(\check{r}), \check{r}\right],
$$

17. According to the this constraint, $\Sigma$ cannot be arbitrarily close to the apparent horizon since that would require the number density $n$ to diverge on $\Sigma$, which takes the dust fluid outside of its regime of validity. We shall impose this constraint in the example that we discuss below.

We now construct an example as follows. We set $8 \pi \bar{\rho}=$ 1 and assume that $0 \leq r \leq 99 / 10$ for the interior region. It follows from Eq. 10 that the collapsing object has a gravitational mass $M=161.7$ and a Bekenstein-Hawking entropy $S_{\mathrm{BH}}=3.3 \times 10^{5}$ for the ensuing black hole in the

16 For some choices of the LTB metric functions, it is possible for the future event horizon to encounter the past curvature singularity at some $r>0$ [see App. A]. It is then implied that the hydrodynamics conditions (4) are violated somewhere on the future event horizon. Additionally, it follows that there are no spacetime slices that fall entirely outside of the future event horizon. Thus, the integral 14 does not account for the entropy of a non-black hole object.

17 The factor 4 is added so that the equality case for the relation 17) results in the entropy 14 being bounded by $S_{\mathrm{BH}}$ 
Planck units. We then require the spacetime to admit a null Cauchy surface $\Sigma$ which is foliated by the spheres with $\dot{R}=0$ in the interior region, i.e. $u=\pi$ everywhere on the interior part of $\Sigma$. Evaluating Eq. (8) at $u=\pi$ we find

$$
R[t(r), r]=\frac{r}{3 k(r)}, \quad t(r)=t_{0}(r)+\frac{\pi}{6 k(r)^{3 / 2}},
$$

the latter giving the defining equation for the surface $\Sigma$ in the interior region. We can then use the fact that $\Sigma$ is a null surface together with Eqs. (5), (8) and (18) to find a differential equation for the bang function $t_{0}(r)$ :

$$
\begin{aligned}
& \frac{d t}{d r}=\left.\frac{R^{\prime}(t, r)}{\sqrt{1-r^{2} k(r)}}\right|_{\Sigma} \\
& \Rightarrow t_{0}^{\prime}(r)=\frac{1}{3 k(r)}\left[\frac{1-\frac{r k^{\prime}(r)}{k(r)}}{\sqrt{1-r^{2} k(r)}}+\frac{3 \pi}{4} \frac{k^{\prime}(r)}{k(r)^{3 / 2}}\right] .
\end{aligned}
$$

Thus, the knowledge of $k(r)$ is sufficient to fully specify the interior geometry. Once we have the interior metric, we can find the location of the future event horizon by first solving Eq. (8) for $r_{\text {out }}$ to find the time $t_{*}$ when the outermost spherical shell becomes trapped, and then solve the following equation for the null geodesic in the interior region with $\left\{t_{*}, r_{\text {out }}\right\}$ as its end point:

$$
\begin{aligned}
& \frac{d t}{d r}=\frac{R^{\prime}(t, r)}{\sqrt{1-r^{2} k(r)}} \\
& =\frac{\partial_{r}\left[\frac{r}{6 k(r)}\left(1-\cos \left[\mathcal{F}\left\{6 k(r)^{3 / 2}\left(t-t_{0}(r)\right\}\right]\right)\right]\right.}{\sqrt{1-r^{2} k(r)}},
\end{aligned}
$$

where we defined $\mathcal{F}(u-\sin u) \equiv u$ and used Eq. 8). We could then confirm that the surface $\Sigma$ is indeed located outside of the future event horizon.

We proceed by finding a differential equation for $k(r)$ by saturating both the non-degeneracy condition given in (4) and the shell physical distance condition given in 17) for some spherical shells on $\Sigma$. On a given constant $t$ surface intersecting $\Sigma$ at $\left\{t_{\Sigma}(\check{r}), \check{r}\right\}$, we have

$$
\begin{aligned}
& \int_{\tilde{r}}^{\check{r}} \sqrt{g_{r r}} d r=\int_{\tilde{r}}^{\check{r}} \frac{R^{\prime}[t(r), r] d r}{\sqrt{1-r^{2} k(r)}} d r \\
& \approx \frac{1}{\sqrt{1-\check{r}^{2} k(\check{r})}} \int_{\tilde{r}}^{\check{r}} R^{\prime}[t(r), r] d r \\
& =\frac{R[t(\check{r}), \check{r}]-2 M[\check{r}]}{\sqrt{1-\check{r}^{2} k(\check{r})}}=R[t(\check{r}), \check{r}] \sqrt{1-\check{r}^{2} k(\check{r})} \\
& =4 \times(8 \pi \rho[t(\check{r}), \check{r}])^{-1 / 4}
\end{aligned}
$$

where we approximated the integral by assuming that the shell at $\{t(\check{r}), \check{r}\}$ is nearly trapped, i.e. $R[t(\check{r}), \check{r}]-$ $2 M(\check{r})=1-\check{r}^{2} k(\check{r}) \ll 1$. We construct $k(r)$ by solving Eq. (21) for $7 \leq r \leq 99 / 10$ and then continuously joining that solution to a slowly varying function of $r$ for $0 \leq r \leq$

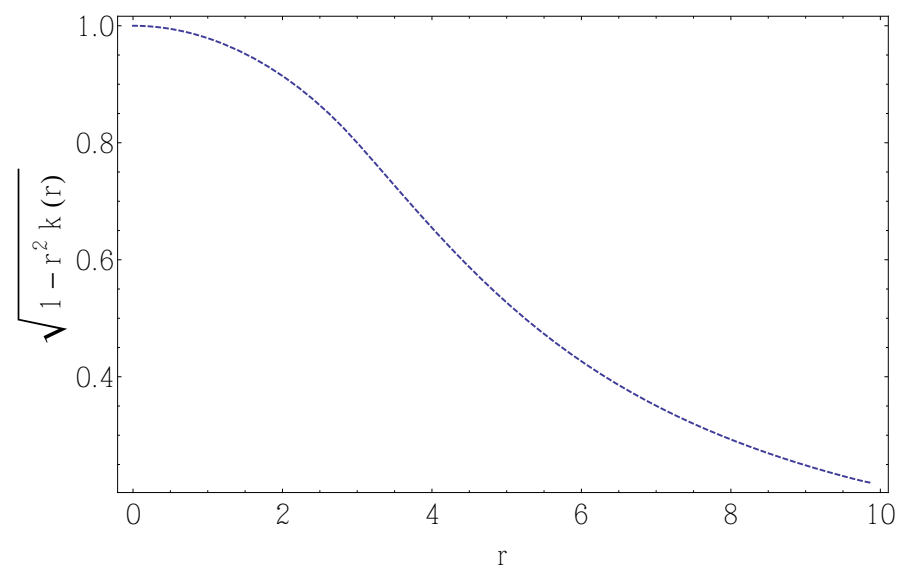

FIG. 1. This is the plot of $\sqrt{1-r^{2} k(r)}$ versus $r$. As expected, $1-r^{2} k(r) \ll 1$ for $7 \leq r \leq 99 / 10$.

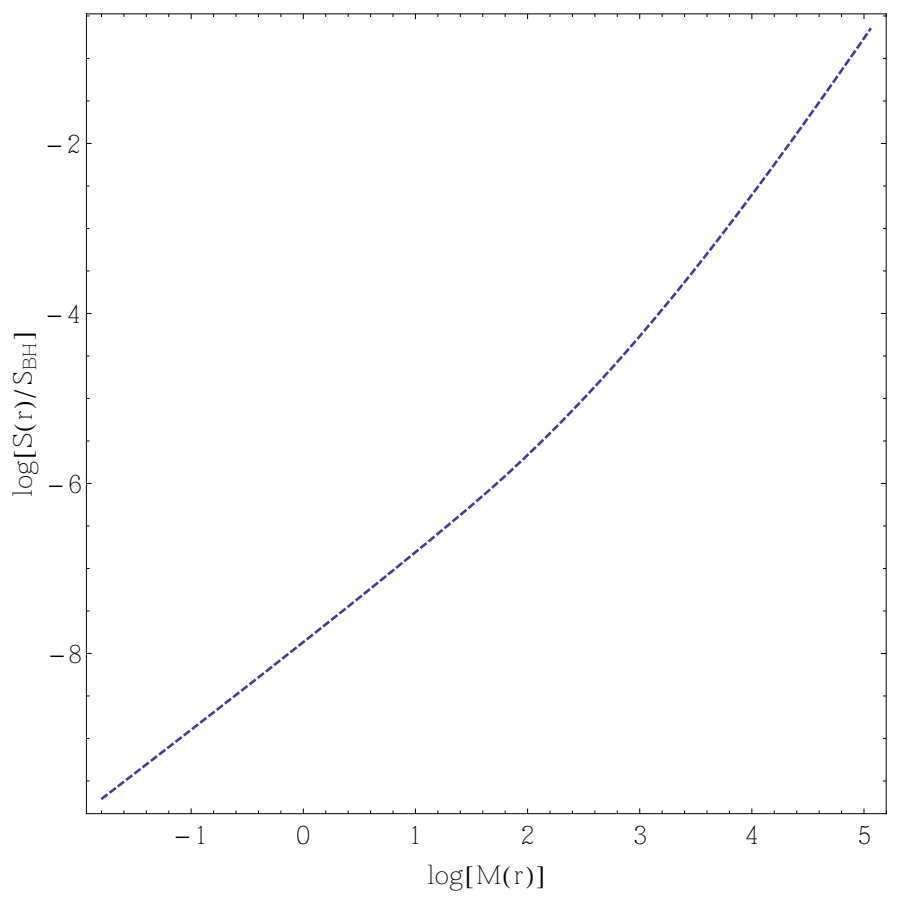

FIG. 2. This is the plot of $\log \left[S(r) / S_{\mathrm{BH}}\right]$ versus $\log [M(r)]$ for the example we have constructed here. Notice that the contribution of the spherical shells at $r \geq 7$ brings the entropy given in Eq. 14 close to the Bekenstein-Hawking entropy $S_{\mathrm{BH}}=3.3 \times 10^{\circ}$.

7 We plot the resulting $\sqrt{1-r^{2} k(r)}$ in Fig 1 . Note that $1-r^{2} k(r) \ll 1$ for $7 \leq r \leq 99 / 10$ is consistent with the assumption $R[t(r), r]-2 M(r) \ll 1$ for these shells used in Eq. (21).

With $k(r)$ in hand, we can now compute the integral

18 The resulting $k(r)$ need not necessarily be $C^{\infty}$ but it should be at least $C^{2}$. 


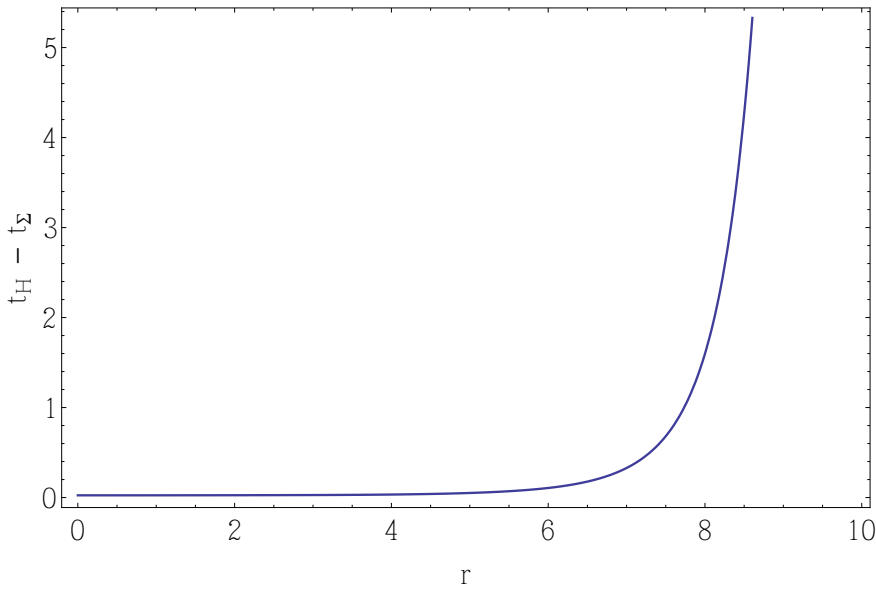

FIG. 3. This is the plot of $t_{\mathrm{H}}-t_{\Sigma}$ versus radius $r$. The future event horizon is indeed to the future of the slice $\Sigma$

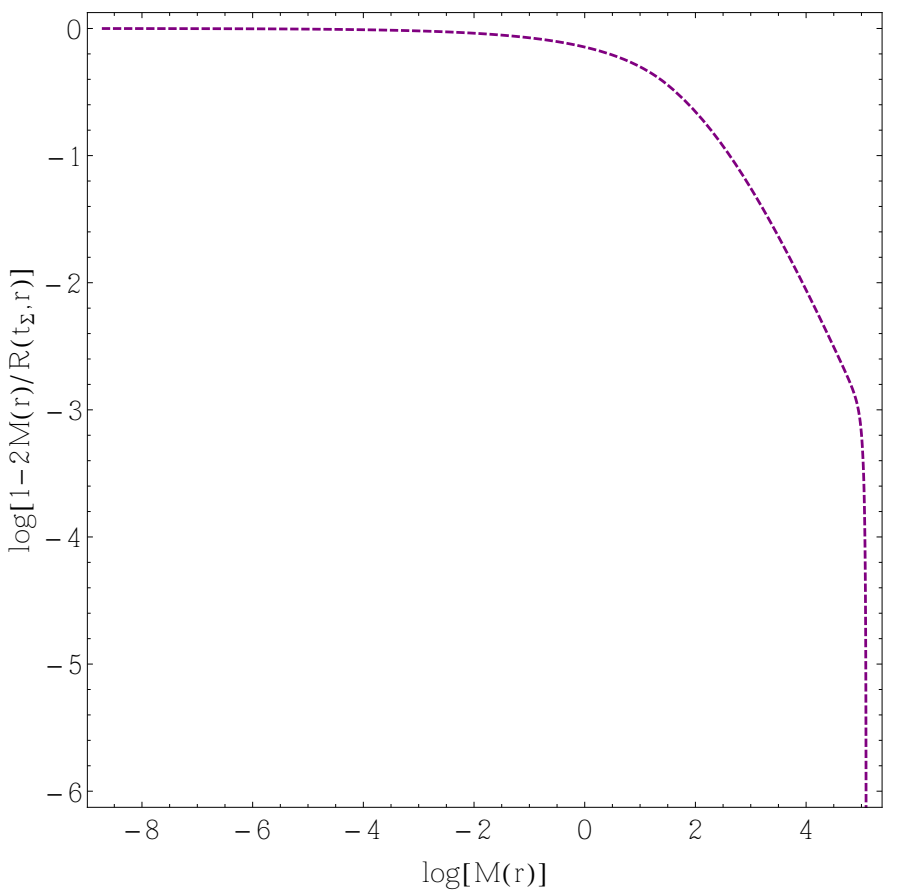

FIG. 4. Here we plot $\log \left[1-2 M(r) / R\left(t_{\mathrm{H}}, r\right)\right]$ verses $\log [M(r)]$ along the future event horizon. Notice that none of the spherical shells are trapped, except for the outermost one for which $\log \left[1-2 M\left(r_{\text {out }}\right) / R\left(t_{\mathrm{H}}, r_{\text {out }}\right)\right]$ diverges.

(14) to find the total entropy

$$
S=1.8 \times 10^{5} \approx 0.55 S_{\mathrm{BH}},
$$

which turns out to be quite close to saturating the Bekenstein-Hawking entropy bound. See Fig. 2

Upon solving Eqs. (19) and (20), we find the location $t_{\Sigma}$ of the slice $\Sigma$ as well as the location $t_{\mathrm{H}}$ of the future event horizon. We plot $t_{\mathrm{H}}-t_{\Sigma}$ in Fig. 3. Note that the surface $\Sigma$ is completely outside of the future event horizon. Additionally, as expected, the future apparent horizon is inside the future event horizon. See Fig. 4.

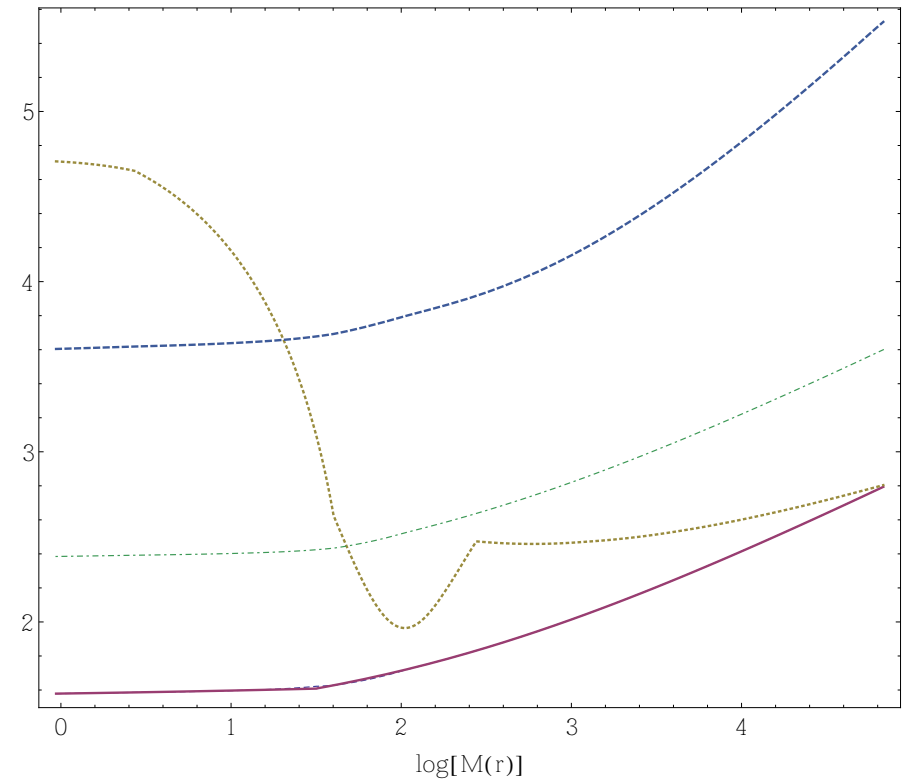

FIG. 5. This is the plot of $\log \left[n^{-1 / 3}\right]$ (the solid blue line), $\log \left[(8 \pi m)^{-1}\right]$ (the solid violet line which is collinear with the solid blue line), $\log \left[(16 \pi / 3)^{1 / 3} m /|\mathbf{D} m|\right]$ (the dotted brown line), $\log \left[\rho^{-1 / 4}\right]$ (the green dotted-dashed line), and log $[\mathcal{L}]$ (the blue dashed line) versus $\log [M(r)]$ along the future event horizon.

Finally, in Fig. 5 we plot the four relevant fluid micro-length scales together with the radius of curvature, $\left\{n^{-1 / 3},(8 \pi m)^{-1},(16 \pi / 3)^{1 / 3} m /|\mathbf{D} m|, \rho^{-1 / 4}, \mathcal{L}\right\}$, defined in Sec. III along the future event horizon. Notice that all micro-length scales are larger than the particle spacing $n^{-1 / 3}$ everywhere along the future event horizon. Notice that the third condition given in (4) is close to becoming saturated. This indicates that the length scales over which the particle mass function $m(r)$ varies is becoming comparable to the particle spacing $n^{-1 / 3}$ along the future event horizon. Also, one should note that all five length scales are above the Planck length.

\section{ATTEMPTS TO FIND PAST GEOMETRIES WITH NO INITIAL SINGULARITY}

In Sec. (III C we constructed a collapsing dust object that saturates the Bekenstein-Hawking entropy bound. However, as we mentioned in the introduction, the global time symmetry of the resulting spacetime renders it pathological due to the presence of a white hole. We find it worthwhile to attempt to construct spacetimes that have the same large entropy but not the white hole singularity in the past.

In the remainder of this paper, we report on a formalism that we have explored in order to circumvent the past white hole singularity. We find it convenient to initially lay out the formalism for the closed FRW geometries, and then apply it to the LTB geometries with $k(r)>0$. As we 
show below, the numerical investigations appear to be indicative of the success of this formalism in both avoiding the white hole singularity and keeping the interior modified spacetime's stress energy tensor from violating the dominant energy condition ${ }^{19}$ However, we show in Sec. IV B that a boundary stress energy tensor is needed to match the interior metric to the exterior Schwarzschild geometry. Given that this boundary stress energy tensor only satisfies the weak energy condition, the overall spacetime only satisfies the weak energy condition as well.

\section{A. The modified closed}

\section{Friedman-Robertson-Walker geometry}

In this section we show that one can modify the closed FRW geometry in the past in order to avoid the white hole singularity. By construction, the stress energy tensor of the modified spacetime satisfies the dominant energy conditions.

The metric for the modified spacetime is

$$
d s^{2}=a(\eta)^{2}\left[-d \eta^{2}+d \chi^{2}+\left(\frac{\sin \sqrt{\kappa(\eta)} \chi}{\sqrt{\kappa(\eta)}}\right)^{2} d \Omega^{2}\right]
$$

where $\eta \equiv \int d t / a(t)$ is the conformal time and $\chi \equiv$ $\int d r / \sqrt{1-\kappa r^{2}}$. Here, unlike the closed FRW geometry, the spatial curvature $\kappa$ is a function of the conformal time $\eta 20$. We require $\kappa(\eta)>0$ at all times and $\kappa(\eta)=1$ for $\eta \geq \eta_{\Phi}$, where $\eta_{\Phi}$ is a conformal time beyond which the spacetime geometry reduces to the closed FRW geometry. For times $-\infty<\eta \leq \eta_{\Phi}$, we define the metric functions $a(\eta)$ and $\kappa(\eta)$ by the following set of equations

$$
\begin{aligned}
& 2 \mathcal{H}^{\prime}+\mathcal{H}^{2}+\kappa\left(1+\frac{\mathcal{L}}{\mathcal{H}}\right)=0, \\
& \mathcal{L}^{\prime}-\frac{1}{2} \mathcal{L}^{2}+\mathcal{H} \mathcal{L}=2 g \mathcal{H}^{\prime},
\end{aligned}
$$

with the initial conditions

$$
\kappa\left(\eta_{\Phi}\right)=1, \quad \kappa^{\prime}\left(\eta_{\Phi}\right)=0, \quad 0<\mathcal{H}\left(\eta_{\Phi}\right) \ll 1 \frac{121}{25}
$$

The functions appearing in Eq. 24 are $\mathcal{L} \equiv \kappa^{\prime} / \kappa, \mathcal{H} \equiv$ $a^{\prime} / a$, and $g(\eta) \equiv 1-\exp \left[-\left(\eta_{\bar{\Phi}}-\eta\right)\right]$ for $\eta \leq \eta_{\bar{\Phi}}$ and zero otherwise. One might consider other choices of $g(\eta)$ depending on the smoothness properties that one wishes to demand of the stress energy tensor. For this particular choice of $g(\eta)$, the stress energy tensor will be continuous at $\eta=\eta_{\bar{\Phi}}$ and smooth everywhere else. Geometrically,

19 See 21] for a definition of the energy conditions.

20 See footnote 10.

${ }^{21}$ Note that we are evolving the metric functions from $\eta_{\Phi}$ to $-\infty$. Therefore, the sign of $\mathcal{H}\left(\eta_{\Phi}\right)$ is positive as the spacetime is expanding in this direction. we start from a point near the moment of time symmetry for the closed FRW geometry and evolve the spacetime backwards in time while diluting the spatial curvature on constant $\eta$ slices. The objective is to push the moment of time symmetry off to $\eta \rightarrow-\infty$, thereby avoiding the white hole singularity.

Before discussing the properties of the modified FRW spacetime, we find it helpful to comment on the motivation behind the set of equations given in (24). The first equation can be regarded as the conservation of the homogenized Misner-Sharp mass. Indeed if we define $R(t, r)=r a(t)$ and require $k$ to have time dependence in Eq. (11, we arrive at the first equation in 24 by setting $M_{, t}=0$. The second equation in 24 is perhaps less motivated, mainly designed to give a sufficiently slow evolution for $\kappa$ in order to keep the modified spacetime from violating the dominant energy condition.

We now provide more details on the solutions to the dynamical equations given in $(24)$. To see that the moment of time symmetry is avoided at any finite time, note that initially $\mathcal{H}^{\prime}\left(\eta_{\Phi}\right)<0$ and $\mathcal{H}\left(\eta_{\Phi}\right)>0$ as we evolve the spacetime backwards in time. If $\mathcal{L}<0$ and $\kappa>0$ at all times $\eta<\eta_{\Phi}$, then $\mathcal{H}$ cannot go to zero since that would imply $\mathcal{H}^{\prime} \rightarrow+\infty$, which is a contradiction.

To see how $\mathcal{L}$ evolves in time, note that the second equation in 24 together with the initial conditions $\mathcal{L}\left(\eta_{\Phi}\right)=\mathcal{L}^{\prime}\left(\eta_{\Phi}\right)=0$ implies that $\mathcal{L}^{\prime \prime}\left(\eta_{\Phi}\right)<0$. Thus, $\mathcal{L}<0$ at times $\eta<\eta_{\Phi}$ and sufficiently close to $\eta_{\Phi}$. Furthermore, we expect to have $\mathcal{L}>-\mathcal{H}$ as long as $\kappa>0$. Indeed if $\mathcal{L}=-\mathcal{H}$ at some finite time $\eta_{*}<\eta_{\Phi}$, Eq. 24) implies $\mathcal{L}^{\prime}\left(\eta_{*}\right)>\left|\mathcal{H}^{\prime}\left(\eta_{*}\right)\right|=\mathcal{H}\left(\eta_{*}\right)^{2} / 2$. Therefore, while $\mathcal{L}$ initially decreases below 0 we must have $\mathcal{L}>-\mathcal{H}$. The only way that $\mathcal{L}$ can become equal to $-\mathcal{H}$ at some time $\eta_{*}$ is to have $0 \leq \mathcal{L}^{\prime}\left(\eta_{*}\right) \leq\left|\mathcal{H}^{\prime}\left(\eta_{*}\right)\right|$ which is inconsistent. Likewise, $\mathcal{L}\left(\eta_{*}\right)=0$ is forbidden since it requires $\mathcal{L}^{\prime} \geq 0$ in the vicinity of $\eta_{*}$, whereas Eq. 24 implies $\mathcal{L}^{\prime}=2 g \mathcal{H}^{\prime}<0$ at $\eta_{*}$.

In fact, given our choice of initial conditions, it is not difficult to see that the dynamical system under study has an attractor solution $\mathcal{L} \rightarrow-\mathcal{H}$. Informally, we can see that this solution is a true attractor by writing $\mathcal{L}(\eta)=$ $\mathcal{L}_{0}(\eta)+\delta(\eta)$, where $\mathcal{L}_{0}=-\mathcal{H}$ and initially $|\delta| \ll\left|\mathcal{L}_{0}\right|$. We can then substitute this expression for $\mathcal{L}$ into the second equation in (24) and assume $\left|\eta-\eta_{\Phi}\right| \gg 1$ to find the following differential equation for $\delta$

$$
\delta_{, \eta}-\frac{1}{2} \delta^{2}-2 \mathcal{L}_{0} \delta=0
$$

Since for the attractor solution we have $\mathcal{H} \approx-\mathcal{L} \approx 2 /|\eta|$, Eq. (26) gives $\delta \propto 1 / \eta^{4}$, which falls off faster than $\mathcal{L}_{0}$ for large $|\eta|$ as expected. Thus, we have $a \propto \eta^{2}$ and $\kappa \propto 1 / \eta^{2}$ as $\eta \rightarrow-\infty$. As a result, one can check that the components of the Riemann tensor for the constant $\eta$ slices go to zero as $\eta \rightarrow-\infty$. This is equivalent to saying that the constant $\eta$ slices become nearly intrinsically flat at large $|\eta|$.

For the dominant energy condition to be satisfied we 


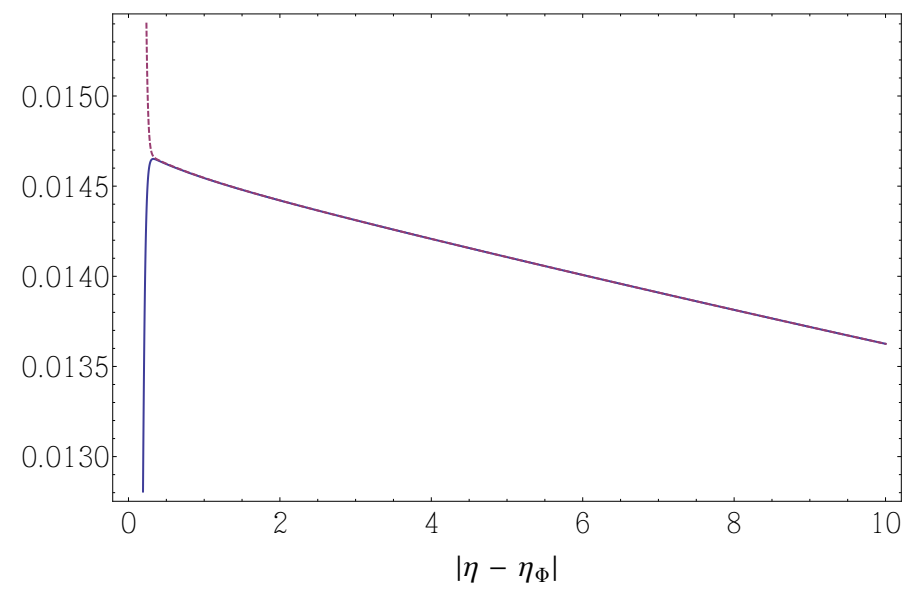

FIG. 6. This is the plot of $|\mathcal{L}|$ (the solid blue line) and $\mathcal{H}$ (the dashed purple line) versus $\left|\eta-\eta_{\Phi}\right|$ as we evolve the spacetime backwards in time. Notice that $|\mathcal{L}|$ converges to $\mathcal{H}$ very quickly.

must have

$$
\rho \geq\left|p_{\hat{x}_{1}}\right|, \quad \rho \geq\left|p_{\hat{\theta}}\right|,
$$

at all times, where $\left\{\rho, p_{\hat{x}_{1}}, p_{\hat{\theta}}\right\}$ are the independent eigenvalues of the stress energy tensor in a locally orthonormal frame

$$
d s^{2}=-d \hat{x}_{0}^{2}+d \hat{x}_{1}^{2}+d \hat{\theta}^{2}+d \hat{\phi}^{2}
$$

( $p_{\hat{\theta}}=p_{\hat{\phi}}$ due to spherical symmetry). Given the metric 23), a routine calculation gives ( $\eta$ dependence is suppressed)

$\rho=\frac{1}{16 \pi a^{2}}\left[G_{\eta \eta}-G_{\chi \chi}+\sqrt{\left(G_{\eta \eta}+G_{\chi \chi}\right)^{2}-4 G_{\eta \chi}^{2}}\right]$,

$p_{\hat{x}_{1}}=\frac{1}{16 \pi a^{2}}\left[-G_{\eta \eta}+G_{\chi \chi}+\sqrt{\left(G_{\eta \eta}+G_{\chi \chi}\right)^{2}-4 G_{\eta \chi}^{2}}\right]$,

$p_{\hat{\theta}}=\frac{\kappa}{8 \pi a^{2} \sin (\sqrt{\kappa} \chi)^{2}} G_{\theta \theta}$

where $G_{a b}$ is the Einstein's tensor. We should first show that the quantity inside the square root in Eq. 29 remains positive semi-definite at all times. This condition is also necessary to ensure that the eigenvector associated with $\rho$ is timelike. It is sufficient to have

$G_{\eta \eta}+G_{\chi \chi} \geq 2\left|G_{\eta \chi}\right|$

$\Rightarrow 2 \mathcal{H}^{2}-2 \mathcal{H}^{\prime}+2 \kappa+\frac{1}{2} \mathcal{L}^{2}\left[\chi^{2} \kappa-f(\chi, \kappa)\right]+f(\chi, \kappa) \mathcal{L}^{\prime}$

$\geq-2 \chi \kappa \mathcal{L}$

$\Rightarrow 2 \mathcal{H}^{2}-2[1-g f(\chi, \kappa)] \mathcal{H}^{\prime}+2 \kappa-f(\chi, \kappa) \mathcal{H} \mathcal{L}+\frac{1}{2} \chi^{2} \kappa \mathcal{L}^{2}$

$\geq-2 \chi \kappa \mathcal{L}$,

where $f(\chi, \kappa) \equiv 1-\chi \sqrt{\kappa} \cot (\chi \sqrt{\kappa})$ and we used the fact that $\mathcal{L} \leq 0$ at all times. We also used Eq. (24) in the last inequality. Since $0<\kappa \leq 1$ and $0 \leq \chi \leq \pi / 2$, we have $0 \leq f \leq 1$. Given that $|\mathcal{L}|<\mathcal{H}$ at all times, the inequality 30 comes down to

$$
\begin{aligned}
& 2 \mathcal{H}^{2}-2[1-g f(\chi, \kappa)] \mathcal{H}^{\prime}+2 \kappa\left[1-\frac{\pi}{2} \mathcal{H}\right]-f(\chi, \kappa) \mathcal{H} \mathcal{L} \\
& +\frac{1}{2} \chi^{2} \kappa \mathcal{L}^{2} \geq 0
\end{aligned}
$$

which is correct at times $\eta<\eta_{\Phi}$ because $\mathcal{H} \ll 1, \mathcal{L} \mathcal{H}<0$, and $[1-g f(\chi, \kappa)] \mathcal{H}^{\prime}<0$, with the latter following from Eq. (24) and $0 \leq g<1$.

To show $\rho \geq\left|p_{\hat{x}_{1}}\right|$, it is sufficient to show that $G_{\eta \eta}-$ $G_{\chi \chi} \geq 0$. Using Eq. 24, we have

$G_{\eta \eta}-G_{\chi \chi}=4 \mathcal{H}^{2}+2[1-g f(\chi, \kappa)] \mathcal{H}^{\prime}-3 f(\chi, \kappa) \mathcal{H} \mathcal{L}+4 \kappa$

$+\mathcal{L}^{2}\left[1-\frac{1}{2} f(\chi, \kappa)-\frac{3}{2} \chi \sqrt{\kappa} \cot (\chi \sqrt{\kappa})\right.$

$\left.+\frac{1}{2} \kappa \chi^{2}\left(-1+\cot (\sqrt{\kappa} \chi)^{2}\right)\right] \geq 4 \mathcal{H}^{2}+2 \mathcal{H}^{\prime}-3 f(\chi, \kappa) \mathcal{H} \mathcal{L}$

$+4 \kappa-\mathcal{L}^{2} \geq 2 \mathcal{H}^{2}+3 \kappa-\kappa \frac{\mathcal{L}}{\mathcal{H}}-3 \mathcal{H} \mathcal{L} \geq 0$.

Finally, we ought to show that $\rho \geq\left|p_{\hat{\theta}}\right|$. As for $\left|p_{\hat{\theta}}\right|$, we have

$$
\begin{aligned}
& 8 \pi a^{2}\left|p_{\hat{\theta}}\right|=\mid-\kappa \frac{\mathcal{L}}{\mathcal{H}}\left[1+\frac{1}{2} g f(\chi, \kappa)\right]-\frac{1}{2} g f(\chi, \kappa)\left[\kappa+\mathcal{H}^{2}\right] \\
& +\frac{1}{2} f(\chi, \kappa) \mathcal{H} \mathcal{L}+\frac{1}{4} \chi^{2} \kappa \mathcal{L}^{2} \mid \leq-\frac{3}{2} \kappa \frac{\mathcal{L}}{\mathcal{H}}+\frac{1}{2}\left[\kappa+\mathcal{H}^{2}\right]-\frac{1}{2} \mathcal{H} \mathcal{L} \\
& +\frac{1}{4} \chi^{2} \kappa \mathcal{L}^{2} \leq 2 \kappa+\mathcal{H}^{2}\left[1+\frac{1}{4} \chi^{2} \kappa\right] \leq 2\left[\kappa+\mathcal{H}^{2}\right] .
\end{aligned}
$$

Likewise we have for the locally measured energy density

$$
\begin{aligned}
& 8 \pi a^{2} \rho=\frac{1}{2}\left[G_{\eta \eta}-G_{\chi \chi}+\sqrt{\left(G_{\eta \eta}+G_{\chi \chi}\right)^{2}-4 G_{\eta \chi}^{2}}\right] \\
& \geq G_{\eta \eta}-\left|G_{\eta \chi}\right|=3\left[\kappa+\mathcal{H}^{2}\right]-2 f(\chi, \kappa) \mathcal{H} \mathcal{L}+\chi \kappa \mathcal{L} \\
& +\frac{1}{2} \mathcal{L}^{2}\left[f(\chi, \kappa)-\frac{1}{2}+\frac{1}{2} \kappa \chi^{2} \cot (\chi \sqrt{\kappa})^{2}\right] \\
& \geq 3\left[\kappa+\mathcal{H}^{2}\right]+\chi \kappa \mathcal{L} \geq\left[3-\frac{\pi}{2} \mathcal{H}\right] \kappa+3 \mathcal{H}^{2}
\end{aligned}
$$

where we used

$$
f(\chi, \kappa)-\frac{1}{2}+\frac{1}{2} \kappa \chi^{2} \cot (\chi \sqrt{\kappa})^{2}>0 .
$$

As can be seen from the last expressions in Eq. (33) and Eq. (34), for $\rho \geq\left|p_{\hat{\theta}}\right|$ it suffices to have $1-(\pi / 2) \mathcal{H} \geq 0$, which is true for $\eta<\eta_{\Phi}$ given that $\mathcal{H} \ll 1$ at such times. Therefore, we conclude that the modified FRW spacetime satisfies the dominant energy condition everywhere to the past of $\eta_{\Phi}$.

\section{B. A numerical study of the modified Lemaître-Tolman-Bondi geometry}

We now apply the formalism developed in Sec. IV A to the LTB metric given in Eq. (5). The equations (24) 
in this case become

$$
\begin{aligned}
& \dot{R}^{2}+2 R \ddot{R}+r^{2} k\left(1+\frac{\dot{k} R}{k \dot{R}}\right)=0, \\
& \dot{R} \frac{\dot{k}}{k}-\frac{1}{2} R\left(\frac{\dot{k}}{k}\right)^{2}+\partial_{t}\left(R \frac{\dot{k}}{k}\right)=2 g \ddot{R},
\end{aligned}
$$

where dot denotes differentiation with respect to the LTB time coordinate $t$ and $g=1-\exp \left[-\left\{t_{\Phi}(r)-t\right\}\right]$. We define $t_{\Phi}(r)$ to be the location of the spacelike slice between $t_{\Sigma}$ and the future event horizon found in Sec. IIIC for which all spherical shells have

$$
\begin{aligned}
& \dot{R}\left[t_{\Phi}(r), r\right] \approx \frac{\left(\frac{\pi}{2}-3 k(r)^{3 / 2}\left[t_{\Phi}(r)-t_{0}(r)\right)\right]}{2} r \sqrt{k(r)} \\
& =-\frac{10^{-6}}{2} r \sqrt{k(r)},
\end{aligned}
$$

where $k(r)$ is the spatial curvature that we constructed in Sec. IIIC The relation given in the first line of Eq. (37) comes from the Taylor expansion of $\dot{R}$ near $t_{\Sigma}$ for which $\dot{R}=0$ everywhere. The numerical factor $10^{-6}$ in the second line of Eq. (37) is chosen so that all spherical shells are located between $t_{\Sigma}$ and the future event horizon.

We numerically solve the coupled system of ordinary differential equations (36) for $-\infty<t<t_{\Phi}(r)$ and $0 \leq$ $r \leq 99 / 10$ using the following initial conditions 22

$$
\begin{aligned}
& k\left[t_{\Phi}(r), r\right]=k(r), \quad \dot{k}\left[t_{\Phi}(r), r\right]=0, \\
& \dot{R}\left[t_{\Phi}(r), r\right]=\frac{10^{-6}}{2} r \sqrt{k(r)} .
\end{aligned}
$$

We then examine the locally measured energy density and principal pressures of the modified spacetime, which are given by

$$
\begin{aligned}
& \rho=\frac{1}{16 \pi}\left[G_{t t}-G_{r}^{r}+\sqrt{\left(G_{t t}+G_{r}^{r}\right)^{2}-4 G_{t r} G_{t}^{r}}\right], \\
& p_{\hat{x}_{1}}=\frac{1}{16 \pi}\left[-G_{t t}+G_{r}^{r}+\sqrt{\left(G_{t t}+G_{r}^{r}\right)^{2}-4 G_{t r} G_{t}^{r}}\right], \\
& p_{\hat{\theta}}=\frac{1}{8 \pi} G_{\theta}^{\theta} .
\end{aligned}
$$

The modified LTB spacetime shares very similar qualitative features to its homogeneous counterpart. In particular, $\dot{k} / k \rightarrow-\dot{R} / R$, or equivalently $R \propto t^{2 / 3}$ and $k \propto t^{-2 / 3}$, at times $t \ll t_{\Phi}(r)$ for all spherical shells. Similar to the case of the modified FRW metric, it is not difficult to check that the constant $t$ slices become nearly intrinsically flat as $t \rightarrow-\infty$. Despite the fact that the eigenvalues of the modified stress energy tensor no longer have the same functional form as the ones obtained in the previous section as they now contain terms with radial derivatives, our extensive numerical investigations provide no evidence for the violation of the dominant energy condition. Therefore, we are convinced that the

\footnotetext{
${ }^{22}$ Recall that we are integrating backwards in time, so $\dot{R}>0$.
}

modified LTB spacetime constructed using (36) avoids the white hole singularity while satisfying the dominant energy condition. In Fig. 7 we plot the eigenvalues of the modified stress energy tensor in a local orthonormal frame for spherical shells at a few different radii.

Finally, note that there is also a non-vanishing boundary stress energy tensor for $t<t_{\Phi}\left(r_{\text {out }}\right)$. This is due to the discontinuity of the time-time component of the boundary extrinsic curvature in this region of spacetime. Indeed, $\vec{u}=\vec{\partial}_{t}$ is no longer a geodesic in the exterior Schwarzschild geometry for $t<t_{\Phi}\left(r_{\text {out }}\right)$, while remaining a geodesic in the interior geometry at all times. The independent eigenvalues of the boundary stress energy tensor in the orthonormal $\{t, \hat{\theta}, \hat{\phi}\}$ frame are given by 23

$$
\bar{\rho}=0, \quad \bar{p}=-\frac{1}{16 \pi} \frac{r^{2} k}{R \sqrt{1-\frac{2 M}{R}+\dot{R}^{2}}} \frac{\dot{k} R}{k \dot{R}},
$$

which are evidently positive semi-definite at all times due to the fact that $(\dot{k} R) /(k \dot{R}) \leq 0$ [See Fig. 6]. Therefore, the boundary stress energy tensor satisfies the weak energy condition.

\section{Black hole's formation timescale}

We find it appropriate to define the black hole's formation timescale to be the interval along $\mathcal{I}_{-}$between the two ingoing null geodesics that intersect the beginning of the event horizon and the outer boundary of the collapsing object as it crosses the event horizon [See Fig. 8. Intuitively, this corresponds to the time required for the collapsing object to cross its own event horizon as measured by the asymptotic null observers at $\mathcal{I}_{-}$.

We now show that this timescale is on the order of $M^{2}$ for the modified LTB collapse models constructed in Sec. IVB To begin, notice that the modified LTB geometry evolves very slowly compared to the LTB geometry. In fact, to a good approximation we have

$$
\Delta v=v_{1}-v_{2}=\Delta \bar{t}+\Delta \bar{r}_{*} \approx \Delta \bar{t},
$$

where $\bar{r}_{*}$ is the Schwarzschild's tortoise coordinate 24 In other words, the proper observer on the boundary is nearly stationary between the times $\bar{t}_{1}$ and $\bar{t}_{2}$. As a result, we have

$$
\Delta \bar{t} \approx \frac{\Delta t}{\sqrt{1-\frac{2 M}{R\left(t_{1}, r_{\text {out }}\right)}}},
$$

where $\Delta t$ is the proper time interval between $\bar{t}_{1}$ and $\bar{t}_{2}$ measured by the proper observer on the boundary. Using the fact that the proper observer on the boundary

\footnotetext{
23 The boundary stress energy tensor comes from a standard calculation for the exterior Schwarzschild geometry. We have also used Eq. [36). See 22].

${ }^{24} \bar{r}_{*} \equiv \bar{r}+2 \bar{M} \log |\bar{r} /(2 M)-1|$
} 

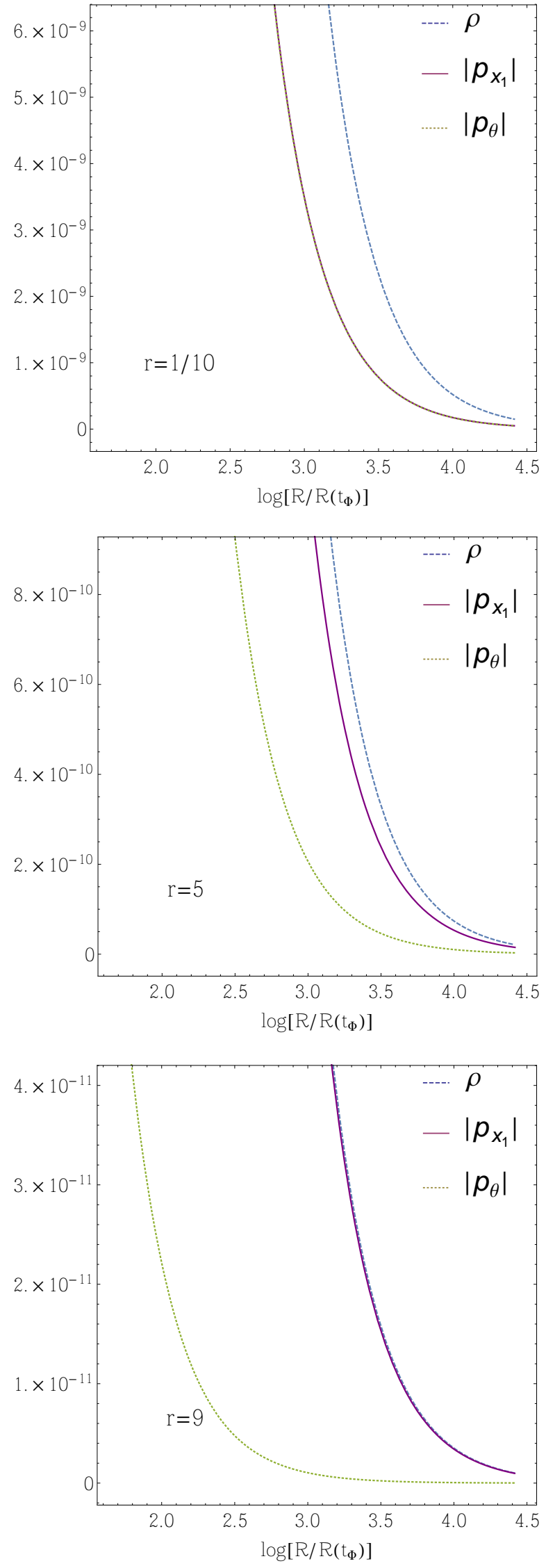

FIG. 7. This is the plot of the eigenvalues of the modified stress energy tensor versus $\log \left[R(t, r) / R\left(t_{\Phi}, r\right)\right]$ for spherical shells at three different radii.

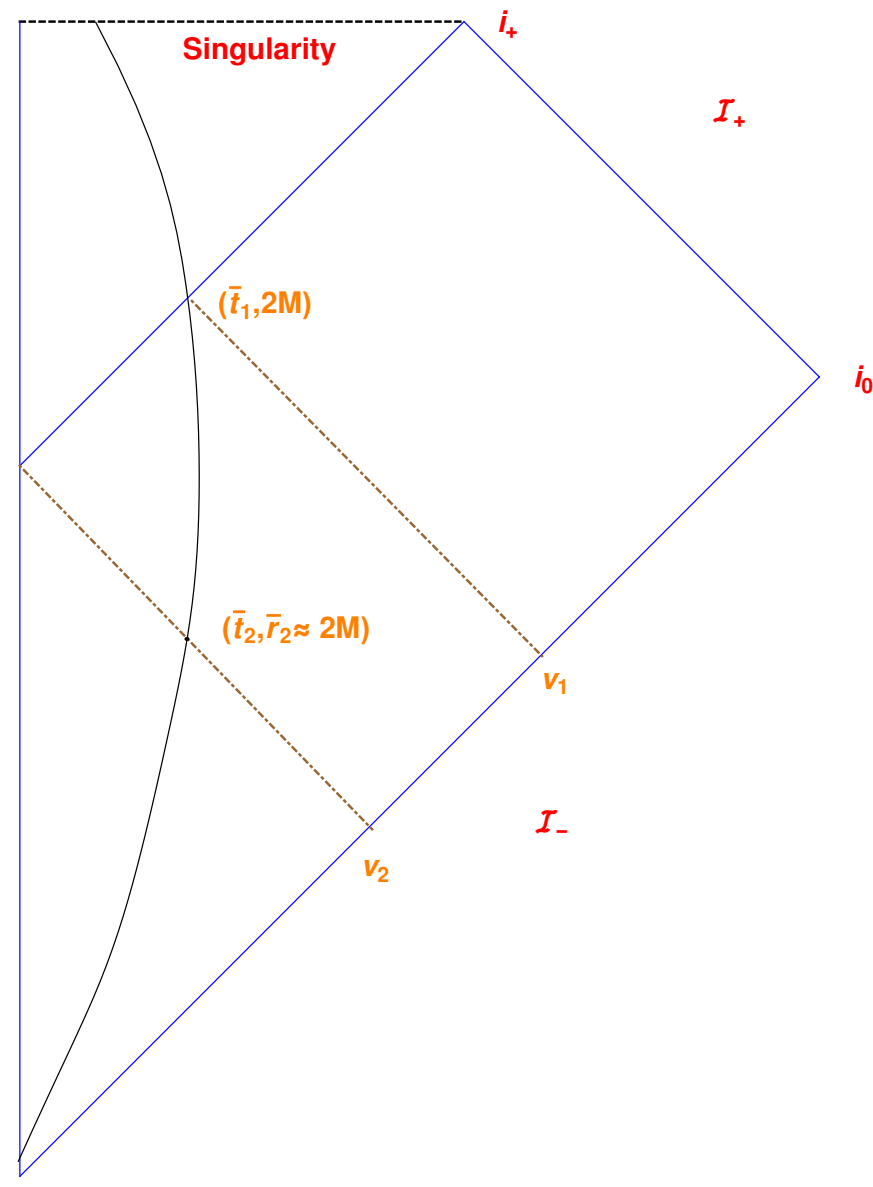

$i_{-}$

FIG. 8. This is the Penrose diagram of a collapsing object embedded in a Schwarzschild exterior. The ingoing null rays with advanced Eddington-Finkelstein coordinates $v_{1}$ and $v_{2}$ intersect the collapsing object at $\left(\bar{t}_{1}, 2 M\right)$ and $\left(\bar{t}_{2}, \bar{r}_{2} \approx 2 M\right)$ respectively.

remains quite close to its initial location where $\dot{R} \approx 0$, it follows from the Eqs. (19), and (21) that

$$
\Delta \bar{t} \sim M \rho_{\text {out }}^{1 / 4} \Delta t \sim M \rho_{\text {out }}^{1 / 2} S \sim M^{3} \rho_{\text {out }}^{1 / 2} \sim M^{2},
$$

where $S$ is the entropy evaluated on $\Sigma$ and $\rho_{\text {out }} \equiv$ $\rho\left[t_{\Phi}\left(r_{\text {out }}\right), r_{\text {out }}\right] \sim r_{\text {out }}^{-6} \sim M^{-2}$, assuming that $k \sim r^{-2}$ for the outer shells [See Sec. III C]. To arrive at (43), we also used the slow evolution of the interior metric to argue that $\Delta t$ is on the order of the temporal change along the future event horizon. We then approximated the temporal change along the event horizon using its value along the surface $\Sigma$. This is a reasonable approximation given the proximity of $\Sigma$ to the event horizon.

\section{CONCLUDING REMARKS}

In this paper we revisited the validity of the conjectured entropy-mass-timescale relation given in (1). We 
constructed a pathology free spacetime from a monsterlike initial condition that saturates the BekensteinHawking entropy bound and forms a black hole within a timescale on the order of $M^{2}$. The constructed spacetime satisfies the weak energy condition. This construction invalidates the conjectured entropy-mass-timescale relation by a factor of $M^{1 / 4}$.

However, the constructed spacetime appears to be finely tuned and probably does not represent a generic scenario of gravitational collapse. Moreover, though beyond the scope of this paper, the question of whether the spacetimes constructed using the recipe provided in Eqs. 24) and (36) are classically and quantum mechanically stable needs to be addressed. Perhaps one way of looking at our conclusion is that violating the conjectured entropy-mass-timescale relation can only be done by some contrived mathematical construction.

Finally, we note that discovering any connection between the relation given in Eq. (1) and the quantum focusing conjecture formulated in 23] can be illuminating.

\section{ACKNOWLEDGMENTS}

We are very grateful to Éanna Flanagan for his comments on the manuscript. We also thank Leonard Gross, Nils Deppe, and Alexander Grant for helpful discussions.

\section{Appendix A: Lemaître-Tolman-Bondi geometry with event horizon intersecting the singularity}

In Sec. III C we mentioned that it is possible for some LTB-Schwarzschild geometries not to have any achronal slices that are completely outside of the future event horizon. It is then implied that in these spacetimes the future event horizon intersects the past curvature singularity. As an example, let us assume that the interior LTB geometry has a radial coordinate $r$ that covers from $r=0$ to $r=99 / 10 \equiv r_{\text {out }}$. Now consider a constant time slice $\Theta$ for which we set $t=0$ and that it is foliated by the maximal spheres, i.e. $\dot{R}\left(t_{\Theta}, r\right)=0$ everywhere on $\Theta$. After setting $8 \pi \bar{\rho}=1$, it follows from Eq. (8) that $u=\pi$ and

$$
t_{0}(r)=-\frac{\pi}{6 k(r)^{3 / 2}}
$$

As for $k(r)$, we take the same solution as in Sec. IIIC by assuming that the non-degeneracy condition (4) the shell-apparent horizon physical distance condition (17) are both saturated. We then solve Eq. 20 to find the location of the future event horizon subject to the appropriate boundary value for $r_{\text {out }}$. It turns out that the future event horizon intersects the past curvature singularity at $r \approx 8.657$. See Fig. 9

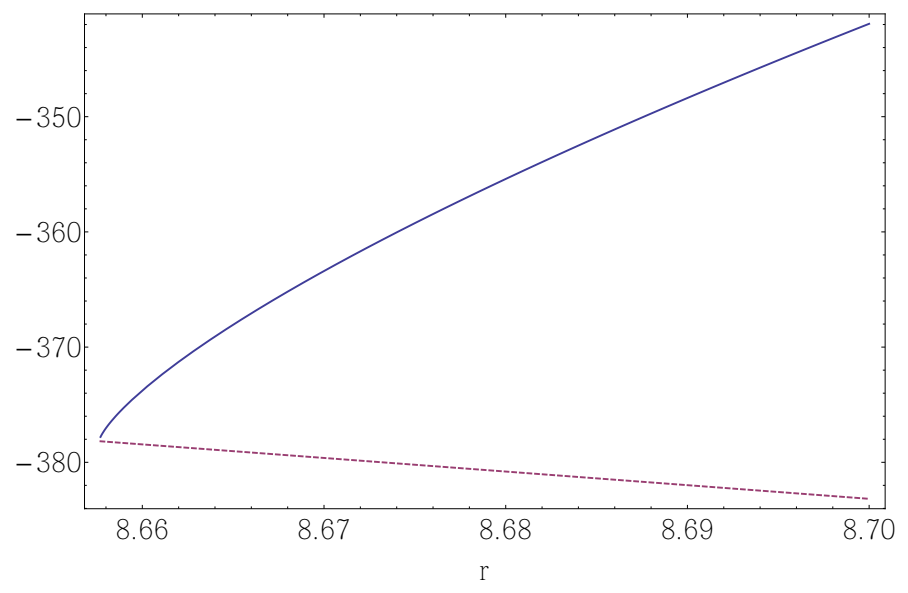

FIG. 9. This is the plot of $t_{\mathrm{H}}$ (solid blue line) and $t_{0}(r)$ (dashed violet line) versus $r$. The future event horizon intersects the curvature singularity at $r \approx 8.657$.

\section{Appendix B: On the validity of the Gaussian normal coordinates for the modified metrics}

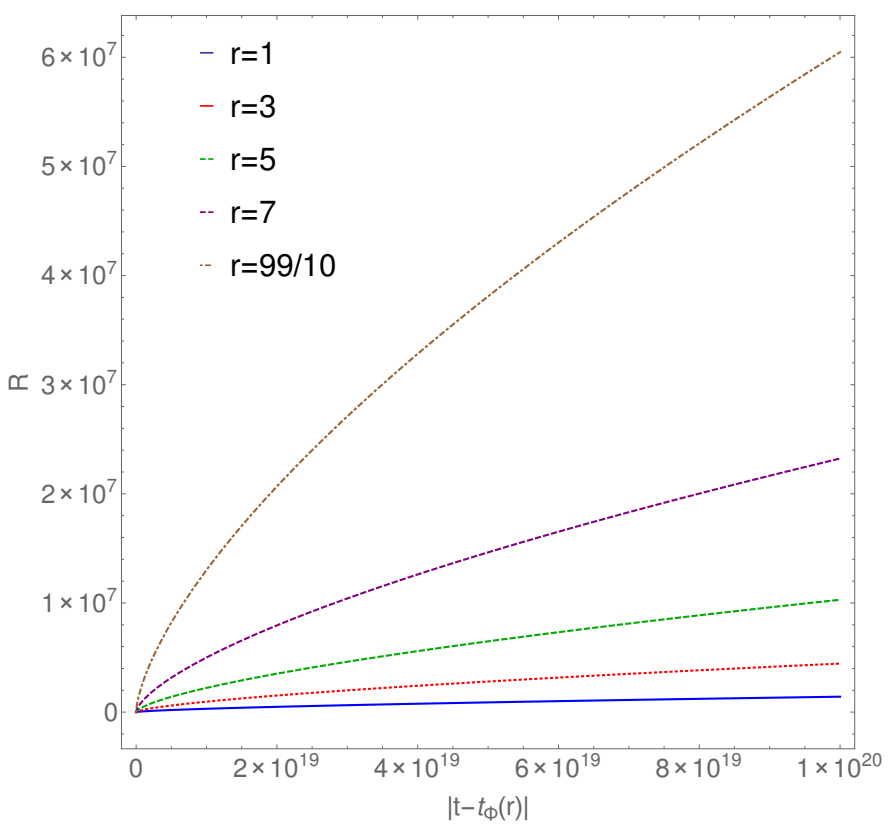

FIG. 10. Here we have plotted the solution of Eq. (36) for a number of shells. As can be seen from the figure, shells expand without any crossing.

In Sec. IV we introduced a formalism in which the radial-radial component of the FRW or LTB metric had an additional time dependence. We implicitly assumed that the Gaussian normal coordinates used to define the FRW and LTB metrics can also be used to define the modified metrics. Here we sketch an argument showing the validity of this assumption.

Recall that the Gaussian normal coordinates can be constructed for a spacetime if and only if there exists 
a family of $C^{n \geq 1}$ timelike geodesics in the spacetime for which every point is located on exactly one of these timelike geodesics. The coordinates are then constructed with $\vec{u}=\vec{\partial}_{t}$ being the unit tangent to each timelike geodesic. A trivial consequence of the existence of such a family of timelike geodesics is that there are no congruences of timelike geodesics with unit tangent $\vec{u}=\vec{\partial}_{t}$ that form a caustic anywhere in the spacetime 25 .

The modified LTB metric is given by

$$
d s^{2}=-d t^{2}+\frac{R^{\prime}(t, r)^{2} d r^{2}}{1-r^{2} k(t, r)}+R(t, r)^{2} d \Omega^{2}
$$

where $r^{2} k(t, r)<1$ at all times and $\dot{k}(t, r) \neq 0$ for $-\infty<t<t_{\Phi}(r)$ [see Sec. IV B. Here we have implicitly assumed that the Gaussian normal coordinates used to define the LTB metric (5) can be used to define the modified LTB metric as well. To ensure that this can be done, we must show that the expansion $\theta \equiv{ }^{(3)} g^{a b} \nabla_{a} u_{b}$ does not diverge for any congruence of timelike geodesics with unit tangent $\vec{u}=\vec{\partial}_{t}$ 26 A short calculation gives

$$
\begin{aligned}
& \theta=\frac{1}{2}{ }^{(3)} g^{a b} \dot{g}_{a b} \\
& =\frac{\dot{R}^{\prime}(t, r)}{R^{\prime}(t, r)}+\frac{\dot{R}(t, r)}{R(t, r)}+\frac{r^{2} \dot{k}(t, r)}{2\left[1-r^{2} k(t, r)\right]} .
\end{aligned}
$$

Pursuant to our discussions in Sec. IV A and IV B with regards to the modified metric functions and their asymptotic behaviour, we do not expect any divergences to occur in (B2) at any radii and time in the past. Therefore, we do not see a problem with the use of Gaussian normal coordinates for the modified FRW and LTB metrics.

\section{Appendix C: Aerial radii do not coincide for distinct coordinate radii in the modified spacetime}

If two aerial radii corresponding to two distinct coordinate radii coincide at some time to the past of $t_{\Phi}(r)$, the formalism of Sec. IV] would be invalidated. Numerically we can confirm that this does not happen for the solutions to the system of equations 36 . See Fig. 10
[1] Jacob D. Bekenstein. Black holes and entropy. Phys. Rev., D7:2333-2346, 1973. doi: 10.1103/PhysRevD.7.2333.

[2] S. W. Hawking. Particle creation by black holes. Comm. Math. Phys., 43(3):199-220, 1975. URL http: //projecteuclid.org/euclid.cmp/1103899181.

[3] S. W. Hawking. Black holes and thermodynamics. Phys. Rev. D, 13:191-197, Jan 1976. doi: 10.1103/PhysRevD.13.191. URL http://link.aps .org/ doi/10.1103/PhysRevD.13.191

[4] Andrew Strominger. Black hole entropy from near horizon microstates. JHEP, 02:009, 1998. doi:10.1088/11266708/1998/02/009.

[5] A. Ashtekar, J. Baez, A. Corichi, and Kirill Krasnov. Quantum geometry and black hole entropy. Phys. Rev. Lett., 80:904-907, 1998. doi: 10.1103/PhysRevLett.80.904

[6] Robert M. Wald. The thermodynamics of black holes. Living Reviews in Relativity, 4(6), 2001. doi: 10.1007/lrr-2001-6. URL http://www.livingreviews . org/lrr-2001-6

[7] W. H. Zurek and Kip S. Thorne. Statistical mechanical origin of the entropy of a rotating, charged black hole. Phys. Rev. Lett., 54:2171-2175, May 1985. doi: 10.1103/PhysRevLett.54.2171. URL http://link.aps . org/doi/10.1103/PhysRevLett.54.2171

[8] Daniel Harlow. Jerusalem Lectures on Black Holes and Quantum Information. 2014.

\footnotetext{
${ }^{25}$ Except at the curvature singularity, which is formally not part of the spacetime.

$26{ }^{(3)} g^{a b}$ is the inverse metric for constant $t$ slices.
}

[9] Gia Dvali and Cesar Gomez. Black Holes as Critical Point of Quantum Phase Transition. Eur. Phys. J., C74:2752, 2014. doi:10.1140/epjc/s10052-014-2752-3.

[10] Rafael D. Sorkin, Robert M. Wald, and Zhen Jiu Zhang. Entropy of selfgravitating radiation. Gen. Rel. Grav., 13: 1127-1146, 1981. doi:10.1007/BF00759862.

[11] Stephen D. H. Hsu and David Reeb. Black hole entropy, curved space and monsters. Phys. Lett., B658:244-248, 2008. doi:10.1016/j.physletb.2007.09.021

[12] Stephen D. H. Hsu and David Reeb. Monsters, black holes and the statistical mechanics of gravity. Mod. Phys. Lett., A24:1875-1887, 2009. doi: 10.1142/S0217732309031624

[13] Douglas M. Eardley. Death of white holes in the early universe. Phys. Rev. Lett., 33:442-444, Aug 1974. doi: 10.1103/PhysRevLett.33.442. URL http://link.aps . org/doi/10.1103/PhysRevLett.33.442.

[14] Robert M. Wald and Sriram Ramaswamy. Particle production by white holes. Phys. Rev. D, 21:2736-2741, May 1980. doi:10.1103/PhysRevD.21.2736. URL http: //link.aps.org/doi/10.1103/PhysRevD.21.2736

[15] Robert M. Wald. Quantum gravity and time reversibility. Phys. Rev. D, 21:2742-2755, May 1980. doi: 10.1103/PhysRevD.21.2742. URL http://link.aps. org/doi/10.1103/PhysRevD.21.2742

[16] H. Bondi. Spherically symmetrical models in general relativity. Monthly Notices of the Royal Astronomical Society, 107(5-6):410-425, 1947. doi:10.1093/mnras/107.56.410. URL http://mnras.oxfordjournals.org/ content/107/5-6/410. abstract

[17] Marie-Noelle Celerier. Do we really see a cosmological constant in the supernovae data? Astron. Astrophys., 353:63-71, 2000.

[18] R. Ali Vanderveld, Eanna E. Flanagan, and Ira Wasserman. Mimicking dark energy with Lemaitre-Tolman- 
Bondi models: Weak central singularities and critical points. Phys. Rev., D74:023506, 2006. doi: 10.1103/PhysRevD.74.023506.

[19] C.W. Misner, K.S. Thorne, and J.A. Wheeler. Gravitation. W.H. Freeman and Company, 1973.

[20] Stephen W. Hawking and G. F. R. Ellis. The Large Scale Structure of Space-Time (Cambridge Monographs on Mathematical Physics). Cambridge University Press, 1975. ISBN 0521099064. URL http://www . amazon.com/ Structure-Space-Time-Cambridge-Monographs-Mathem a dp/0521099064

[21] Robert M. Wald. General Relativity. University Of Chicago Press, first edition edition, 1984.
ISBN 0226870332. URL http://www.amazon.com/ General-Relativity-Robert-M-Wald/dp/0226870332\% 3FSubscriptionId\%3D192BW6DQ43CK9FN0ZGG2\%26tag\% 3Dws $\% 26$ linkCode $\% 3$ Dxm2 $\% 26$ camp $\% 3 D 2025 \% 26$ creative $\%$ 3D165953\%26creativeASIN\%3D0226870332.

[22] Eric Poisson. A relativist's toolkit : the mathematics of black-hole mechanics. Cambridge, UK: Cambridge University Press, 2004.

[23] Raphael Bousso, Zachary Fisher, Stefan Leichenauer, and Aron C. Wall. A Quantum Focussing Conjecture. 2015. 